\title{
Zur Kenntnis der Oszillation physikalischer Konstanten in Homologen-Reihen.
}

\author{
Von Harmany Pauty. \\ Mit einem Nachwort von Johannes StakK. \\ Mit 7 Figuren im Text.
}

Die auffällige Erscheinung des Hinundherschwankens der Konstanten bei homologen organischen Verbindungen ist kürzlich Gegenstand einer Erörterung zwischen G. TAMMANN ${ }^{1}$ ) und E. J. CuY ${ }^{2}$ ) gewesen. Tammans nimmt an, daß das Hinundhergehen der Schmelzpunkte der normalen ein- und zweibasischen Säuren der Paraffinreihe, wie es 1877 von BAEYEr festgestellt worden war, darauf zurückzuführen sei, daß bei paarer Anzahl von Kohlenstoffatomen zwei stabile, bei unpaarer nur eine Kristallart auftritt, und stütźt diese Ansicht durch den Hinweis auf die Zustandsdiagramme der Ameisensäure und Essigsäure. Den Grund ihrer Verschiedenheit sieht er in einer Verschiedenheit des molekularen Aufbaues der betreffenden Flüssigkeiten. E. J. CUY versucht eine elektronische, polare Deutung unter Hinweis auf die Befunde Mesaweins ${ }^{3}$ ) bei der Pinakolinumlagerung. Ich selbst habe früher auch einmal eine ähnliche Vermutung geäuBert ${ }^{1}$ ), erkenne aber heute. daß es auf Grund der neueren Anschauungen der Kristallphysik nicht richtig ist, Erscheinungen der innermolekularen Bindung zur Deutung von Vorgängen an der zwischenmolekularen Bindung, wie sie sich z. B. beim Schmelzen von Kristallen vollziehen, zu benutzen. ${ }^{5}$ ) Das Reißen eines Kraft-

1) Z. anorg. u. allg. Chem. 109 (1920), 221; 115, 288.

2) Z. anorg. u. allg. Chem. 115 (1921), 273.

3) Ann. d. Chem. 419 (1921), $121-175$.

4) Ann. d. Chem. 383 (1911), 260 Anm.

$\left.{ }^{5}\right)$ Deswegen sind such die ähnlichen Erklärungsversuche von BLACH, $Z$. f. physik. Chem. 50 (1905), 61, sowie von Falk und NrLson, Journ. prakt. Chem. 88 (1913), 101 nicht haltbar. 
feldes der zwischenmolekularen Bindung durch thermisch-molekulare Stöße vollzieht sich im allgemeinen ohne wesentliche Änderung der innermolekularen Beziehungen. ${ }^{1}$ )

Eine Bezugnahme auf die deutlich polaren Verhältnisse bei den Fällen Meerweins durch E. J. CUY muß deswegen als nicht glücklich bezeichnet werden, soweit nicht wirklich, wie bei der Dissoziationskonstante, Polarität im Spiele ist. Aber auch da genügen seine Deutungen nicht. Wäre die Polarität bei den Oszillationen der Schmelzpunktswerte von entscheidendem Einfluß, so müBte man erwarten, daß sehr verschieden stark polar wirksame Gruppen auch sehr verschieden starke Schwankungen der Schmelzpunkte veranlaßten bei den einzelnen Reihen. Es ist ein leichtes, an Hand der weiter unten folgenden Diagramme (Fig. 1) sich davon zu überzeugen, daß bei den Schmelzpunkten polar stark verschiedene Substituenten häufig ungefähr gleich starkes Pendeln hervorrufen, z. B. in den Reihen der Glykole, Diamine und Fettsäuren, oder der Dinitrile und Dicarbonsäuren, deren (nicht aufgeführte) Amide noch stärkere Schwankungen zeigen als die Säuren selbst, u. a. m.

Ich selbst habe mich schon seit längerer Zeit mit der Frage nach dem Wesen des fraglichen Problems befaBt und bin nicht auf Grund des Stoffes von $\mathrm{BracH}^{2}$ ) allein, der E. J. Cur hauptsächlich als Unterlage gedient zu haben scheint, zu der Ansicht gekommen, daB die Erscheinungen auch schon bezüglich des Tatsächlichen verwickelter liegen, als man bisher anzunehmen scheint. Es sei mir daher gestattet, meine Befunde und Ansichten darüber mitzuteilen. Ich möchte nicht verfehlen zu erwähnen, daB ich mich bei meinen Studien der wertvollen Beratung seitens meiner hiesigen Kollegen, den Herren JoH. Stark und Hans v. HaLbaN, zu erfreuen hatte. Beiden sei für ihr freundliches Interesse herzlichst gedankt.

Als ein erstes Erfordernis stellte sich die Notwendigkeit heraus, den vorhandenen, von Biach bis 1904 gesammelten Stoff zu überprüfen, zu sichten und zu ordnen. Denn seine Zusammenstellung ist zwar sehr vollständig, enthält aber manche Reihen von zweifelhaftem Werte; mehrfach sind sogar die Werte so unrichtig, daB die Reihen als Belegmaterial wertlos wurden bei der Verbesserung.

Ich habe aber davon abgesehen, das seitdem weiter bekannt gewordene Material vollständig nachzutragen, weil mir mehr daran

1) Vgl. JoH. Stark, Die Prinzipien der Atomdynamik, Die Elektrizität im chem. Atom III, § 23 (Leipzig 1915).

2) Z. f. physik. Chem. 50 (1904), 43. 
lag, die Grundlagen der Erscheinungen aufzudecken und zu zergliedern, als nur den Stoff zu sammeln. Von neuem bringe ich vielmehr nur das, was zum Beleg nützlich ist.

Was nun die Auswahl und Verwertung des Tatsachenmaterinls im allgemeinen anbetrifft, so sei folgendes bemerkt:

1. Scheint es mir nicht ratsam, Reihen von drei und vier Konstanten als Beweis für das Abwechseln zu verwenden. Solche unter fünf bis sechs Werten halte ich für zu leicht der Zufälligkeit unterworfen. Bei längeren Reihen dürfen aber keinesfalls Ausnahmen oder Gegensätze vorkommen, wenn sie Wert haben sollen.

2. Halte ich es für bedenklich, zweite oder gar dritte Differenzen der Werte als Nachweis zu verwenden. Dafür sind doch die Beobachtungsfehlergrenzen im allgemeinen zu groß. Ich werde in dieser Besprechung möglichst nur solche Reihen berücksichtigen, deren Werte selbst schon das Wechseln erkennen lassen.

3. Wurde von mehreren Werten das Mittel der einander zunächst liegenden meisten genommen, und möglichst auch Beobachtungsreihen desselben Urhebers berücksichtigt. Die Auswahl der richtigen zusa mmengehörigen Werte ist natürlich stark Glückssache.

4. Wurde darauf gesehen, möglichst - hinsichtlich der Kohlenstoffkette - einfache Verbindungen zu betrachten, also weniger beispielsweise Ester oder Anhydride, wie freie Säuren, da in jenen zwei Ketten enthalten sind, die die Dinge zu verwickeln scheinen. Das hat den Vorteil, dab es eher möglich ist, auf der nämlichen Unterlage verschiedene physikalische ÄuBerungen miteinander zu vergleichen, denn als am leichtesten und vollständigsten zugänglich sind naturgemäB die einfachsten Körper bäufiger und ausgedehnter untersucht, als ihre Abkömmlinge.

Die Verteilung des Stoffes auf die einzelnen Äußerungsgebiete ist sehr ungleich. Mit Sicherheit kommen bisher in Betracht: Schmelzpunkt, Siedepunkt, Löslichkeit, Molekularvolumen, Molekularwärme, Dissoziationskonstante, Reaktionsgeschwindigkeit ${ }^{x}$ ), optische Drehung; die übrigen von BIAcB aufgeführten sind $\mathrm{m}$. E. unsicher und bedürfen erst weiterer Ausarbeitung.

A. Bei weitem die meisten und klarsten Fälle weist derSchmelzpunkt auf. Das folgende, die Reihen der wichtigsten einfacheren Verbindungen der Fettreihe aufweisende Diagramm läBt ersehen,

1) Vgl. hierzu auch die während des Druckes erschienene Abhandlong von K. SPIRo „ק-Oxydation und parige Bindung", Helv. Chim. Acta IV, 4 (1921), 459. Z. nnorg. u. allg. Chem, Bd. 119. 
da $B$ die Erscheinung der Alternation bier eine recht allgemeine, vielleicht überhaupt eine regelmäßige ist.

Das Diagramm erlaubt folgende Feststellungen für den Schmelzpunkt unverzweigter einfacher (in bezug auf die Anzahl der vorhandenen Kohlenstoffketten) Verbindungen abzuleiten:

a) gerade Kohlenst offzahl bedingt den höheren Schmelzpunkt;

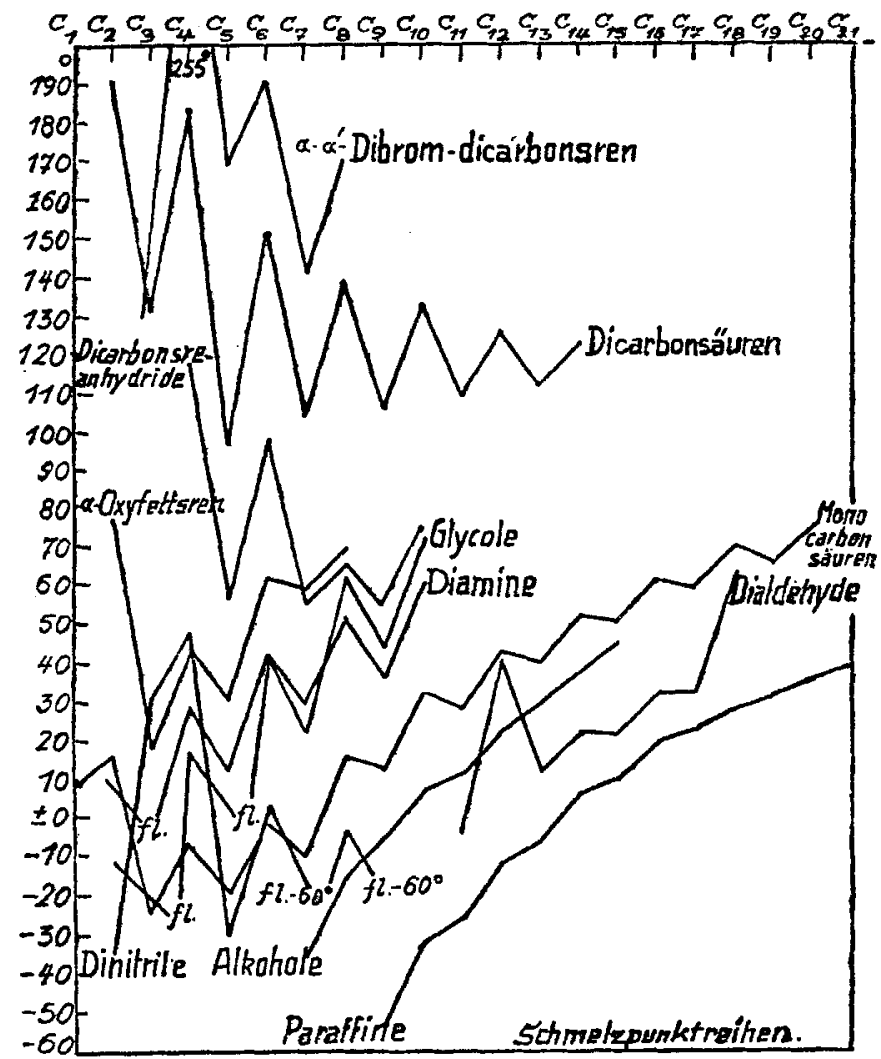

Fig. 1.

b) die Stärke des Pendelns der Werte vermindert sich mit Verlängerung der Kette;

c) Ersatz endständiger Wasserstoff- durch Sauerstoffoder Stickstoffatome steigert das Ausschlagen, besonders wenn der Ersatz beiderseits und unter mehrfacher Bindung stattfindet;

d) Ersatz in 2-Stellung durch Halogen oder Hydroxyl ändert den Verlauf der mittleren Kurve nur unbedeutend; 
e) bei einfach gebundenen Endatomen steigt letztere, bei beiderseits mehrfach gebundenen fällt sie ab.

Diese Regeln können betreffs des letzten Punktes e) noch dahin ergänzt werden, da $B$ der Phenylrest im Sinne einer ungesättigten Gruppe wirkt, wie die Reihe der Benzoylaminoalfaryle zeigt: der Schmelzpunkt geht herunter.

$\begin{array}{lc}\mathrm{C}_{6} \mathrm{H}_{5} \mathrm{CONH} . \mathrm{CH}_{2} \cdot \mathrm{C}_{6} \mathrm{H}_{5} & \text { Schmp. } \\ \mathrm{C}_{6} \mathrm{H}_{5} \mathrm{CONH} \cdot\left(\mathrm{CH}_{2}\right)_{2} \cdot \mathrm{C}_{6} \mathrm{H}_{5} & 116 \\ \mathrm{C}_{8} \mathrm{H}_{5} \mathrm{CONH} \cdot\left(\mathrm{CH}_{2}\right)_{3} \cdot \mathrm{C}_{6} \mathrm{H}_{5} & 57 \\ \mathrm{C}_{6} \mathrm{H}_{5} \mathrm{CONH} \cdot\left(\mathrm{CH}_{2}\right)_{4} \cdot \mathrm{C}_{6} \mathrm{H}_{5} & 83 \\ \mathrm{C}_{6} \mathrm{H}_{5} \mathrm{CONH} \cdot\left(\mathrm{CH}_{2}\right)_{5} \cdot \mathrm{C}_{6} \mathrm{H}_{5} & 60 \\ \mathrm{C}_{6} \mathrm{H}_{5} \mathrm{CONH} .\left(\mathrm{CH}_{2}\right)_{6} \cdot \mathrm{C}_{6} \mathrm{H}_{5} & 62\end{array}$

In merkwürdigem Gegensatz zu Halogen und Hydroxyl (d) steht als Substituent Alkyl, und zwar insbesondere Methyl, insofern es, namentlich in der 2-Stellung ${ }^{1}$ ), offenkundig die Zickzacklinie der Konstanten streckt, wie es die folgenden Schmelzpunktsreihen der homologen alkylierten Dicarbonsäuren im Vergleich mit den nicht alkylierten ersichtlich machen:

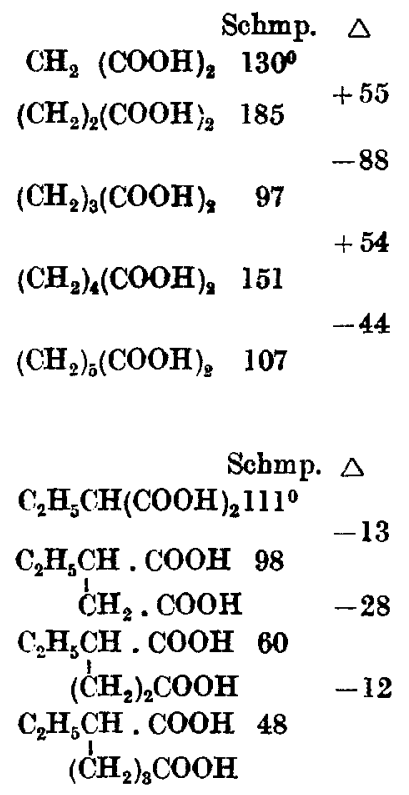

\begin{tabular}{|c|c|c|}
\hline $\mathrm{CH}_{3} . \mathrm{CH} / \mathrm{CO}$ & $\begin{array}{c}\text { Schmp } \\
135^{\circ}\end{array}$ & \\
\hline $\mathrm{CH}_{3} \cdot \mathrm{CH} . \mathrm{COOH}$ & 112 & \\
\hline $\begin{array}{r}\mathrm{CH}_{2} \cdot \mathrm{COOH} \\
\mathrm{CH}_{3} \cdot \mathrm{CH} \cdot \mathrm{COOH}\end{array}$ & 78 & \\
\hline $\mathrm{CH}_{3} \cdot \stackrel{i}{\left(\mathrm{CH}_{2}\right)_{2} \mathrm{COOH}}$ & 63 & \\
\hline $\begin{array}{r}\left(\mathrm{CH}_{2}\right)_{3} \mathrm{COOH} \\
\mathrm{CH}_{3} \cdot \mathrm{CH} \cdot \mathrm{COOH}\end{array}$ & & \\
\hline
\end{tabular}

1) Auf die besondere Bedeutung der 2-Stellung bezüglich der Änderung physikalischer Eigenschaften macht auch H. KAUfFuann (Bezz. zwischen phys. Bigg. und chem. Konstitution, Stuttgart 1920) wiederholt aufmerksam, z. B. S. 34 u. a. m. 
Bei der Bestimmung der Höhe des Schmelzpunktes wirkt bei diesen Reihen das Alkyl jedoch nur untergeordnet mit. Das geradlinig verkettete Grundskelett der Dicarbonsäure behauptet sich vielmehs in dem Sinne, daß seine geraden Kohlenstoffatomzahlen den höheren Schmelzpunkt bedingen, ungeachtet, ob die Zahl der in der Seitenkette vorhandenen und damit die gesamte Kohlenstoffatomzahl gerade oder ungerado ist. ${ }^{1}$ ) Findet sich aber weniger Sauerstoff als in den Dicarbonsäuren vor, wie es bei den Metbylketonen und Dimethyldiketonen der Fall ist, die man vom Standpunkt des Carbonylkohlenstoffatoms aus betrachtet (im Vergleich mit Aldehyden und Dialdehyden oder ein- und zweibasischen Fettsäuren) als verzweigt ansehen kann,

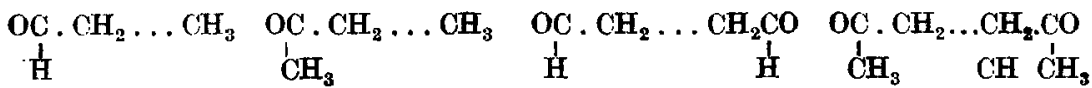

so äußert sich die verflachende Wirkung des Methyls gegenüber der Carbonylgruppe so stark, da $b$ das Pendeln ganz verschwindet. Mir wenigstens jst es nicht möglich, aus der von $\mathrm{BIACH}^{2}$ ) angeführten Reihe der Methylketone ein solches zu erkennen. Auch bei den Dimethyldiketonen kann ich dies nicht, doch zeigt diese, von Genanntem nicht angeführte Reihe auf Beobachtungsfehlern beruhende Unrichtigkeiten, weswegen ich sie ebenfalls hier fortlasse.

Doppelbindungen grenzen nämlich gewissermaßen eine Kette ab. Daher erfolgt ein Hinundherwechseln bei den Schmelzpunkten auch dann, wenn z. B., wie bei den Hexensäuren, eine Doppelbindung vom einen Ende des Kohlenwasserstoffrestes zum anderen wandert.

$$
\begin{array}{lc}
\mathrm{CH}_{3}-\mathrm{CH}_{2}-\mathrm{CH}_{2}-\mathrm{CH}=\mathrm{CH}-\mathrm{COOH} & \begin{array}{c}
\text { Schmp. } \\
\mathrm{CH}_{3}-\mathrm{CH}_{2}-\mathrm{CH}=\mathrm{CH}-\mathrm{CH}_{2}-\mathrm{COOH}
\end{array} \\
\mathrm{CH}_{3}-\mathrm{CH}=\mathrm{CH}-\mathrm{CH}_{2}-\mathrm{CH}_{2}-\mathrm{COOH} & \pm 0 \\
\mathrm{CH}_{2}=\mathrm{CH}-\mathrm{CH}_{2}-\mathrm{CH}_{2}-\mathrm{CH}_{2}-\mathrm{COOH} & \text { fl. }\left(-18^{\circ}\right)
\end{array}
$$

Auch hier haben die so entstehenden geradzahligen Ketten die hochliegenden Schmelzpunkte. Doch ist ein solches Material wegen der unklaren Frage, ob die „Homologen" auch stereochemisch zur gleichen Reihe gehören, so interessant es an sich wäre leider nur von beschränktem Werte abgesehen von der Kürze der Reihe.

Aus diesen Darlegungen verhältnismäßig noch einfacher Fälle sieht man wohl schon, daB es vorerst keinen Zweck hat, weitere Verflechtungen von Ketten zu betrachten. Anstatt weitere Klarheit

1) Dies deutet die schon von Com, Monatsh. f. Ch. 26 (1905), 89 gemachte vermeintliche Feststellung der „Umkehrung“ des Pendelns in diesen Fällen.

2) A. a. 0 . 
zu gewinnen, wird man eher vielleicht Gefahr laufen, in unübersichtliches Gelände zu geraten. Dazu ist, wie ich mich überzeugt habe, auch der Stoff größtenteils noch zu lückenhaft. Vielmehr scheint mir das Vorhandene immerhin wohl schon zu reichen, die grundsätzliche Frage nach der Ursache der Erscheinungen aufzuwerfen.

Zunächst ist nun darauf hinzuweisen, da B das Pendeln keinesfalls kristallmorphologisch, etwa verursacht durch Allotropie ${ }^{1}$ ), gedeutet werden kann. Denn erstens geht mit dem regelmäßigen Wechseln nach meinen Befunden kein Wechseln der Kristallform, wie man denken könnte, einher, sondern man findet oft dieselbe Kristallform bei Gliedern der ungeraden und der geraden Reihe, z. B. bei den zweibasischen Säuren; zweitens müBte dann das Alternieren natürlich auf die Schmelzpunkte beschränkt sein. Da $B$ dies nicht der Fall ist, ist schon durch Brach, wie erwähnt, festgestellt und von E. J. Cuy besonders noch einmal hervorgehoben vorden.

Ganz sicher darf man aber auch sagen, da $B$ in additiven Eigenschaften der homologen Reihen nicht die Ursache für das Pendeln gefunden werden kann. Es ist nicht einzusehen, wie Verbindungen, die sich nur durch die Art der Zahlenreihe bestimmter in ihnen enthaltener, regelmäBig sich wiederholender Gruppen voneinander unterscheiden, hinsichtlich der additiven Eigenschaften voneinander verschieden sein könnten. Für das Atomvolumen jedenfalls kann man sofort zeigen, da.B es nicht in Betracht kommt, indem nach der bekannten Koppschen Berechnung die Werte für Methyl und Brom nahezu gleich groß sind (27,5 gegen 27,8); sie müßten also als Substituenten von gleicher Wirkung sein. Tatsächlich sind sie, wie wir sahen, wesentlich darin verschieden.

In Wirklichkeit können nur, wie man aus dem bisher Besprochnen ohne weiteres schon schließen konnte, konstitutive Gründe für das Oszillieren zar Erörterung gestellt werden.

Aus den oben angeführten Folgerungen b), c) und e) ${ }^{2}$ ) kann man weiter erkennen, daß die Beziehungen der endständigen Gruppen in einer normalen Kette zueinander sehr wahrscheinlich maßgebend sind, weil die Wirkung mit Verlängerung der Kette sich vermindert, und, daB deren Zusammensetzung von Wichtigkeit ist.

1) Vor Tammann (a. a. O.) hat auch Nennst in seinem Lehrbuche über theoretigche Chemie (7. Aufl.) auf diese Möglichkeit (S. 359) hingewiesen.

2) S. 274 und 275. 
Es kommt also an

1. auf die gegenseitige Entfernung der Endgruppen');

2. auf deren Bindungsweise (mehrfache Bindung wirkt stärker und auch anders als einfache);

3. auf die Natur der in den Endgruppen vorhandenen Atome.

4. Folgt aus verschiedenen Umständen, besonders aber aus der besonderen Stellung abgezweigter Alkyle gegenüber anderen Substituenten, die bedeutende Rolle des Baues der Kohlenstoffkette.

Diese Dinge werden weiterhin beleuchtet durch die Konstantenreihe des von Lossen ${ }^{2}$ ) berechneten Molekularvolumens, aus der bei den einfachen Ketten, z. B. denen der n-Fettsäuren offenkundig hervorgeht, $d a ß$ diejenigen mit gerader Kohlenstoffatomzahl die größere Raumerfüllung besitzen.

\begin{tabular}{rrc} 
& $\mathrm{M} .-\mathrm{V}$. & \multicolumn{1}{c}{$\Delta$} \\
$\mathrm{HCOOH}$ & 41,2 & $\mathbf{2 2 , 6 ^ { \circ }}$ \\
$\mathrm{CH}_{3} \mathrm{COOH}$ & $\mathbf{6 3 , 8}$ & 22,0 \\
$\mathrm{C}_{2} \mathrm{H}_{5} \mathrm{COOH}$ & 85,8 & 22,4 \\
$\mathrm{C}_{3} \mathrm{H}_{7} \mathrm{COOH}$ & 108,2 & 21,7 \\
$\mathrm{C}_{4} \mathrm{H}_{8} \mathrm{COOH}$ & 129,9 & 22,7 \\
$\mathrm{C}_{5} \mathrm{H}_{11} \mathrm{COOH}$ & 152,6 & 21,6 \\
$\mathrm{C}_{6} \mathrm{H}_{13} \mathrm{CO} \mathrm{H}$ & 174,2 & 23,4 \\
$\mathrm{C}_{7} \mathrm{H}_{15} \mathrm{COOH}$ & 197,6 &
\end{tabular}

Obwohl die Differenzen in dieser Reihe nicht sehr groß sind, ist doch zu beachten, daß diese aus acht Gliedern besteht, und daB die Werte ganz regelmäßig hin- und herschwanken. Ich halte sie daher für zuverlässig.

Beim Vergleich der Werte des Molekularvolumens mit denen der Schmelzpunkte fällt auf, daß bei den Fettsäuren dem relativ kleineren Molekularvolumen derniederere Schmelzpunkt entspricht.

Fragen wir uns nun, wie wir denn die Verschiedenheit der Raumerfüllung der Homologen mit gerader und ungerader Zahl von $\mathrm{CH}_{2}$ Gruppen uns vorzustellen haben, so haben wir die vorhin aus dem Tatsa.chenmaterial der Schmelzpunkte gezogenen Schlüsse in Überlegung zu ziehen, die alle auf den Bau der Kohlenstoffkette in der Molekel als wichtigstes Moment hinweisen. Da aber vom rein

1) Als Endgruppen sind allgemeiner betrachtet auch solche zu verstehen, die durch eine mehr oder weniger lange Kette von $\mathrm{CH}_{2}$ Gruppen voneinander getrennt sind; bei Verzweigungen kann also eine ,Endgruppe" auch an einem mittleren Kohlenstoffatom hängen.

2) BraCH, a. a. O. 
strukturellen Gesichtspunkt aus doch gar kein Unterschied zwischen Gerade und Ungerade abgeleitet werden kann, so sehe ich zurzeit keine andere Mögliehkeit, als eben entsprechend der Oszillation der Molekalarvolumina räumliche Verhältnisse zu berücksichtigen, d.h. zu versuchen, die Pendelungen sterisch abzuleiten.

Dem mögen die folgenden Ausführungen dienen.

Baxyer hat bekanntlich auf die Bedeutung des Tetraederwinkels von $109^{\circ} 28^{\prime}$ für die Form von Kohlenstoffreihen aufmerksam gemacht. Dieser Winkel bedingt, daß ein drittes Atom, das sich an zwei bereits miteinander verbundene anschlieBt, im genannten Winkel zum ersten sich stellt. Ein weiteres, viertes, hat verschiedene Möglichkeiten. Die Extreme dieser sind, daß entweder es die Polyederform fortsetzt oder sich abgewandt vom ersten Atom stellt, so daß eine Zickzackform der Kette erscheint. Wenn es im ersteren Falle nicht zum Schluß eines z. B. fünfgliedrigen Ringes kommt, würde eine Spiralform sich herausbilden können. Dies hätte zur Folge, daB in so gestalteten Ketten sich ähnliche Beziehungen, wie bei Ringen ergäben, nämlich, daß zwischen den Konstanten der Homologen mit den Kohlenstoffpaaren $1-2,1-5$, sowie $1-3$, 1-4, und ferner 1-6-11, 2-7-12 usw., indem bei einer Spirale diese Atome übereinander in etwa parallel zur Zylinderachse laufenden Linien des Mantels angeordnet wären, gewisse Differenzierungen zutage treten müßten, was indessen bei unseren Fällen nicht erkennbar ist. Denkt man sich statt dessen die Tetraeder in ständig sich gerade fortsetzender, gleichförmiger Weise aneinander gereiht, so hat man, zunächst in der Ebene, rein geometrisch betrachtet, folgende einfachste Möglichkeiten:

I.

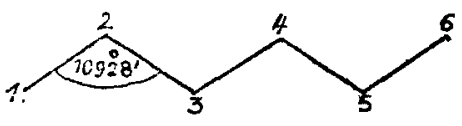

Fig. 2.
II.

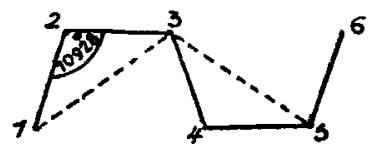

Fig. 3.

Von diesen entsteht die zweite aus der ersten dadurch, da $B$ in dieser jedes zweite Winkelstück ( $3,4,5$ usw.) um $180^{\circ}$ in der Verbindungsachse des zweiten und dritten Atoms gedreht wird. Im Raume kann diese Drehung gemäß den bisher gültigen, formalen Vorstellungen anch um jeden beliebigen anderen Winkelbetrag vor sich gehen, so daB II nur in der Ebene als eine ausgesprochen von I verschiedene Figur erscheint. 
Die Anschauung, daß einfach gebundene Tetraeder um ihre Verbindungsachse fessellos drehbar seien, kann indessen nur für nackte Kohlenstoffatomketten gelten. Sind die an dem mittleren Atomen noch vorhandenen beiden Haftstellen der Tetraeder mit gleichnamigen fremden Atomen, z. B. des Wasserstoffs, besetzt, so orientieren diese die Lage der Tetraeder zueinander, indem sie das Bestreben haben, sich möglichst symmetrisch und zugleich in größtem Abstand voneinander anzuordnen. Das ist, wie man sich am Modell überzeugen kann, am vorzüglichsten gerade bei der Anordnung der Tetraeder in der Ebene, wie in Fig. 2 der Fall. Fig. 3 erscheint noch zulässig, stellt aber keine sehr günstige Konfiguration mehr dar. Eine Kettenanordnung, bei der dagegen auch noch ein fünftes Atom in einem nach innen gerichtetem Winkel (offene Pentagonstellung) sich befinden würde, bevor der nächste Winkel entgegengesetzt sich stellte, wäre dagegen äußerst unwahrscheinlich, da dit zusammengedrängten Wasserstoffgruppen dies nicht zulassen würden. (Eine solche Anordnung könnte nur durch RingschluB stabil werden.)
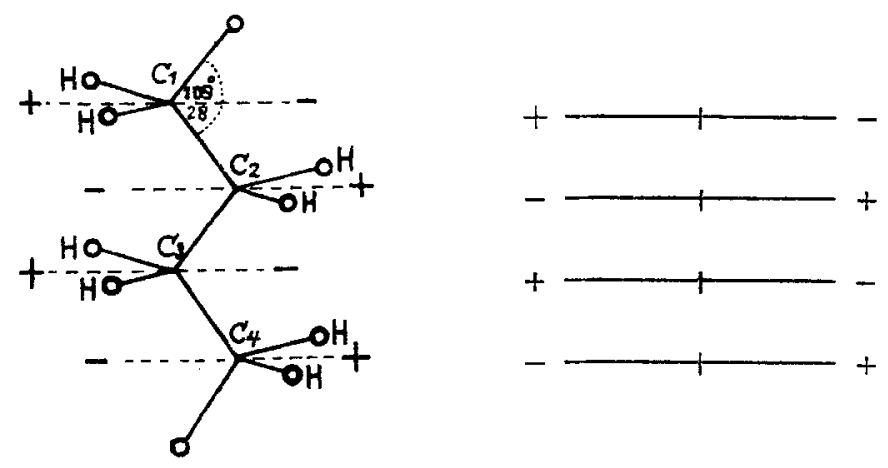

Fig. 4.

Die polare Orientierung durch die beiden Wasserstoffatome der $\mathrm{CH}_{2}$-Gruppe ist ebenfalls durch den Tetraederwinkel bedingt, der sie räumlich einseitig zur Hauptrichtung der Kohlenstoffkette festlegt. Man kann diese Art von Ordnung der $\mathrm{CH}_{\mathbf{2}}$-Gruppen im Raume, die man als polar-sterisch bezeichnen könnte, am besten mit der Orientierung von Magnetnadeln vergleichen und erläutern, indem man annimmt, daß die beiden ,positiven" Wasserstoffatome dem an sich indifferenten Kohlenstoffatom eine positive Seite geben, ähnlich, wie der Stahl durch Streichen eine solche erhält. Eine unmagnetisch aufgehängte Stahlnadel ist um ihre Achse beliebig drehbar, magnf- 
tisiert stellt sie sich ein in bezug auf einen anderen Pol. Würde man mehrere magnetisierte Nadeln übereinander so anordnen, $\mathrm{da} B$ sie alle in derselben Achse drehbar sind, so würden sie sich alle in dieselbe Ebene stellen, aber ihre positiven Pole abwechselnd nach rechts und links richten. Das tertium comparationis wird man aus der vorstehenden perspektivischen Figur 4 sofort 'erkennen.

So wird wohl ganz deutlich, daß die obige Form I (Fig. 2) die jedenfalls statistisch bevorzugte ist. Man begreift aber auch die leicht erfolgende Störbarkeit ihrer Normallage, beispielsweise durch Seitenabzweigung (Methyl); das Gleichgewicht kann so nur in einer anderen, weniger regelmäBig gebauten Konfiguration sich einstellen.

Daß sich ceteris paribus zwischen den Molekeln mit gerader und ungerader Atomzahl der Kette ein Unterschied hinsichtlich des Volumens derart ergeben muß, daß die Summe der Entfernungen zwischen den änßersten Atomen bei den geradzahligen größer ist. als bei den ungeradzahligen, läßt sich leicht durch Messung am Modell feststellen. ${ }^{1}$ ) Auch würde der Umstand, da९ die endständigen Gruppen bei den geradzahligen in einer Art von .,Anti"-Stellung, bei den ungeradzahligen dagegen in einer Art von ,Syn"-Stellung sich befinden, mit der eingangs nachgewiesenen Bedeutung der endständigen Gruppen und ihrer Entfernung voneinander für die Oszillationserscheinung bestens in Einklang sich befinden. Bei der starken Abhängigkeit der im Kristall geordnet liegenden zwischenmolekularen Bindungen von dem Bau und der Anordnung der innermolekularen Bindungen kann es nicht wundernehmen, wenn gerade „polarsterische" Verhältnisse sich in einer großen Empfindlichkeit der zwischenmolekularen Bindungen gegen thermisch-molekulare Stöße, d. h. beim Schmelzvorgang äußern.

Im Gegensatz dazu wird der Siedepunkt, indem es sich hier um die Zerreißung völlig ungeordneter, die Kohäsion veranlassender zwischenmolekularer Bindungen handelt, lediglich durch räumliche Struktur bedingte feinere Unterschiede weniger zutage treten lassen.

Hier findet man nun häufig bei den ungeradzahligen Homologen nach BIAchs Tabellen den höheren Siedepunkt, was mit der

1) Mehr noch, als in den dargelegten struktur-formalen Verschiedenheiten ist der eigentliche Untersehied zwischen Gerade und Ungerade in Krümmungs verschiedenheiten der Ketten zu suchen, wie Jor. Stark in den anhängenden "Folgerungen" darlegt. 
von H. Kauffmann ${ }^{1}$ ) gemachten Ermittelung, daB mit dem kleineren Molekularvolumen der höhere Siedepankt einhergeht, übereinstimmt. Ich führe als Beleg wieder die Fettsäuren an, deren Molekularvolumina vorhin schon wiedergegeben waren:

\begin{tabular}{|c|c|c|}
\hline & Sdp. $760 \mathrm{~mm}$ (ZANDFR) & $\Delta$ \\
\hline $\begin{array}{r}\mathrm{H}-\mathrm{COOH} \\
\mathrm{CH}_{3}-\mathrm{COOH} \\
\mathrm{C}_{2} \mathrm{H}_{5}-\mathrm{COOH} \\
\mathrm{C}_{8} \mathrm{H}_{7}-\mathrm{COOH} \\
\mathrm{C}_{4} \mathrm{H}_{2}-\mathrm{COOH} \\
\mathrm{C}_{5} \mathrm{H}_{11}-\mathrm{COOH} \\
\mathrm{C}_{6} \mathrm{H}_{13}-\mathrm{COOH} \\
\mathrm{C}_{7} \mathrm{H}_{12}-\mathrm{COOH} \\
\mathrm{C}_{8} \mathrm{H}_{12}-\mathrm{COOH} \\
\mathrm{C}_{9} \mathrm{H}_{12}-\mathrm{COOH}\end{array}$ & $\begin{array}{l}100,8^{0} \\
118,1 \\
140,9 \\
162,4 \\
185,4 \\
204,7 \\
223,5 \\
237 \\
254 \\
270\end{array}$ & $\begin{array}{l}17,3 \\
22,8 \\
21,5 \\
23,0 \\
19,3 \\
17,8 \\
13,5 \\
17 \\
16\end{array}$ \\
\hline
\end{tabular}

Hinsichtlich der Löslichkeit müssen wir hingegen wieder deutlichere Unterschiede beim Pendeln der Konstanten finden. Erfahrungsgemäß zeigt sie ja auch eine ähnlich große Empfindlichkeit, aber in entgegengesetztem Sinne (antibat) ${ }^{2}$ ) bei Änderungen in der Molekel wie der Schmelzpunkt.

Auch hier kann die Reihe der Fettsäuren ein geeignetes Beispiel liefern. Allerdings nicht in Form der bei den niederen Gliedern schwer bestimmbaren freien Säuren selbst, sondern in Gestalt ihrer, wohl am ersten normale Verhältnisse zeigenden Silbersalze. ${ }^{3}$ )

$\begin{array}{ccc}\text { Iöslichkeit in } 100 \mathrm{~T} \text {. Wasser }\left(20^{\circ}\right) & \Delta \\ \mathrm{CH}_{3} \mathrm{COOAg} & \mathbf{1 , 0 3 7} & \mathbf{0 , 2 9 1} \\ \mathrm{C}_{2} \mathrm{H}_{5} \mathrm{COOAg} & \mathbf{0 , 8 3 6} & \mathbf{0 , 3 5 2} \\ \mathrm{C}_{3} \mathrm{H}_{7} \mathrm{COOAg} & \mathbf{0 , 4 8 4} & \mathbf{0 , 1 8 4} \\ \mathrm{C}_{4} \mathrm{H}_{9} \mathrm{COOAg} & \mathbf{0 , 3 0 0} & \mathbf{0 , 2 0 0} \\ \mathrm{C}_{5} \mathrm{H}_{11} \mathrm{COOAg} & 0,100 & 0,045 \\ \mathrm{C}_{6} \mathrm{H}_{13} \mathrm{COOAg} & 0,055 & \end{array}$

Gleich den Siedepunkten sind auch die Moleknlarwärmen antibat in Hinsicht auf die Oszillation der Konstanten zum Molekularvolumen. Die Erscheinung des Hinundhergehens der Konstanten ist zuerst von W. Hertz ${ }^{4}$, an den Fettsäuren (fl.) erkannt worden, so daB deren - von mir auf Grund von Zahlen des Schrifttums verlängerte - Reihe hier von neuem als Beispiel dienen kann.

\footnotetext{
ग) A. a. O. S. 28.

2) R. LUTHER, Z. f. Elektrochem. 12 (1908), 97.

3) LaNdolt-BörNsTerN, Tabellen 1912, S. 537.

•) Chem. Ztg. 41 (1917), 183.
} 


\begin{tabular}{|c|c|c|}
\hline & M. W. & $\Delta$ \\
\hline$\underset{\mathrm{CH}}{\mathrm{HCOOH}}$ & 24,5 & 6,8 \\
\hline $\begin{array}{l}\mathrm{CH}_{3} \mathrm{COOH} \\
\mathrm{C}_{2} \mathrm{COOH}\end{array}$ & 31,3 & 8,7 \\
\hline $\mathrm{C}_{8} \mathrm{H}_{7} \mathrm{COOH}$ & 46,3 & 6,3 \\
\hline $\mathrm{C}_{4} \mathrm{H}_{9} \mathrm{COOH}$ & 60,2 & 13,9 \\
\hline $\mathrm{C}_{5} \mathrm{H}_{21} \mathrm{COOH}$ & 61,8 & 1,6 \\
\hline $\mathrm{C}_{1} \mathrm{H}_{12} \mathrm{COOH}$ & 72,6 & $\begin{array}{r}10,8 \\
5.7\end{array}$ \\
\hline $\mathrm{C}_{7} \mathrm{H}_{15} \mathrm{COOH}$ & 78,3 & 12,9 \\
\hline$\underset{\mathrm{C}_{8} \mathrm{H}_{10} \mathrm{C}_{14} \mathrm{COOH}}{\mathrm{H}_{1}}$ & $\begin{array}{l}91,2 \\
90,0\end{array}$ & 1,2 \\
\hline
\end{tabular}

(Die Differenzen scheinen mit Vergrößerung der Molekel größer za werden.)

Optische Drehung der Acyl-1-Menthole und Dissoziationskonstante der Fetţăuren.

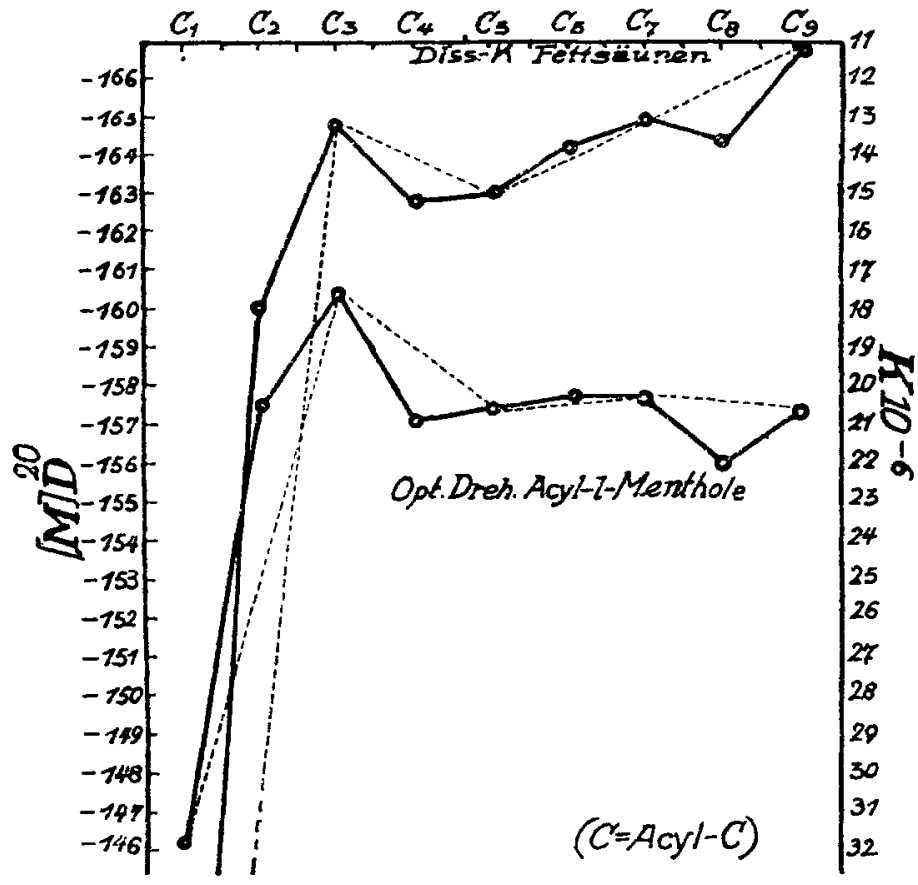

Fig. 5.

B. Der Besprechung verbleiben jetzt noch die Oszillationen der Konstanten der elektrischen Dissoziation und der optischen Drehung. Es ist dies einem gesonderten Abschnitt vorbehalten worden, weil sich hier wichtige Abweichungen von den bisher behandelten Formen des Pendelns der Konstanten zeigen, die bisher nicht erkannt worden waren. Diese treten nicht überall hervor, z. B. nicht bei den zwei- 
basischen Säuren und ihren Abkömmlingen, die die gewöhnliche Form: hoch-niedrig, hoch-niedrig zeigen. Dagegen findet man sie bei den Dissoziationskonstanten der einbasischen n-Fettsäuren und der optischen Drehung solcher Ester von diesen, in denen der Acylrest unmittelbar mit dem am asymmetrischen Kohlenstoffatom hängenden Hydroxylsauerstoffatom verbunden ist. Wie man aus der obigen Figur 5 ersehen kann, in der die Dissoziationskonstanten der Fettsäuren $\left.{ }^{1}\right)$ und die molekularen Drehungen der AcvI-l-Menthole ${ }^{2}$ ) reziprok zu jenen eingetragen sind, spiegeln sich die Größen jener in denen der Drehung fast genau wider.

Die Reziprozität beweist, daß erstens bei den homologen Mentholestern die Konstitution der Acylreste sozusagen das Einzige ist, worin sich die Homologen auch in optischer Hinsicht voneinander unterscheiden, und zweitens, daß im Sinne der Regel von TschíGAEFF „desto bedeutender die optische Wirkung eines inaktiven Substituenten ist, je näher er sich zu einem asymmetrischen Komplex befindet". Denn, wenn der Dissoziationsgrad grob ist, und sich somit ein länger gestrecktes Valenzfeld zwischen dem Kation und dem Anion befindet, bedingt die Länge dieses gleichen Feldes, da $B$ der gleiche Anionrest schwächer zur optischen Wirkung konimt, als im nmgekehrten Falle. Die von der Konstitution der Kohlenwasserstoffreste abhängige Länge des Anionrestfeldes wird als optisches Inkrement ganz belanglos, wenn die Reste nicht mehr mit dem asymmetrischen Kohlenstoffatom selbst verbunden, sondern durch ein dazwischen liegendes Atom von diesem getrennt sind. Es tritt dann, wie es bei den Fettsäureestern des aktiven 1-Amralkohols der Fall ist, nur eine gewöhnliche, einfache Oszillation von dem Aussehen der früher besprochenen Fälle ein. ${ }^{\mathbf{3}}$ )

Bei den in Fig. 5 wiedergegebenen Konstantenreihen liegt nämlich, um es gleich zu sagen, doppelte Oszillation vor, ein Pendeln einer bereits pendelnden Reihe. Um dies leichter an den Diagrammen $\mathrm{zu}$ erkennen, kann man sie gewissermaßen auf eine

1) E. Franke. Z. f. physik. Chem. 16 (1895), 463.

2) L. TSchưgaEm, Ber. 31 (1898), 360, 1775; Compt. rend. (1902), II, 1238. Die vollkommene Analogie der Figuren in dem Diagramm beweist die Fehlerfreiheit beider Linien, da deren Konstanten natürlich ganz unabhängig voneinander festgestellt worden sind. (Die Beachtung der Pendelungen gestattet somit, die Richtigkeit von Konstantẹnreihenzu überprüfen.)

${ }^{3}$ ) Von einer Darstellung des Diagramms der von BIACH bereits aufgeführten Werte kann abgesehen werden. 
Normalform bringen, indem man die Stüeke der punktierten, die Übersicht erleichternden Hilfslinie gleich lang, aber unter Beibehaltung der Winkel, in denen sie zueinander liegen, zugrundelegt, also die Entfernungen der ungeraden Werte voneinander gleich groß wählt, und nun die dex geraden proportional den früheren Verhältnissen einträgt. Man bekommt dann folgendes (wieder umgekehrt dargestellte) Bild der Fettsäure-Dissoziationskonstanten (Fig. 6a):

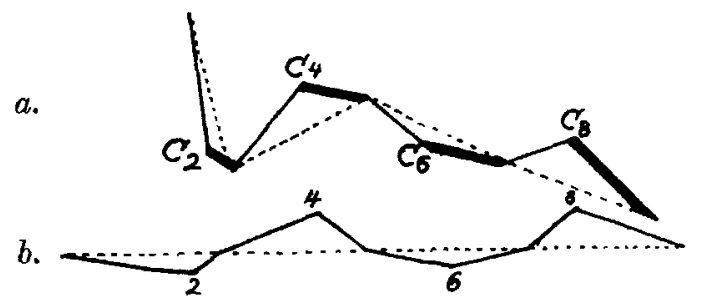

Fig. 6.

In diesem sind die geraden Punkte numeriert, und auBerdem ist durch einen dicken Strich ihre Verbindungslinie mit dem nächsten ungeraden Punkt hervorgehoben. So sieht man ohne weiteres, daB in bezug auf den letzteren die geraden Werte immer oben liegen. Ferner entspricht jedem Punkte nicht, wie bei der einfachen Oszillation der übernächste dritte, sondern erst, wie auch $H$. KaurrMANN ${ }^{1}$ ) gesehen hat, der fünfte. Die Form ist aus einer einfachen Zickzacklinie derart entstanden, daß jedes zweite der mit einer geraden Zahl anfangenden Paare hinaufgesetzt wurde, bzw. jedes erste hinunter. Darin besteht eben die Pendelung einer bereits vorhandenen. Als charakteristisch für doppelte Oszillation ist zu betrachten, daß zwei Mittellinien eingezeichnet werden können, eine für die untere, und eine für die obere pendelnde Reihe.

Würde man nun die punktiert eingezeichnete Hilfslinie strecken, so daB a uf einer Geraden gleiche Abstände der ungeraden Werte lägen, und dann wieder die geraden Werte entsprechend, wie oben eintragen, so würde eine im ersten Augenblick vielleicht etwas seltsame, aber bei näherem Zusehen ganz regelmäßige Form einer Linie erscheinen (in Fig. $6 b$ unterhalb der besprochenen (a) dargestellt). ${ }^{2}$ )

1) A. a. 0 .

$\left.{ }^{2}\right)$ Aus der interessanten Form erkennt man, daB bei den Fettsäuredissoziationskonstanten die Doppeloszillation nicht die einzige Besonderheit darstellt. Man wird bemerken, $\mathrm{da} \beta$ noch zwei andere Erscheinungen vorliegen, nämlich erstens, daß die Abweichungen der unteren geraden Pankte (2,6) von der 
Würden in dieser die geraden Zahlen statt durch gebrochene durch gerade Linien gleichartig verbunden, so hätten wir eine Verbindungslinie von der Form, wie bei der einfachen Pendelung, doch besteht jetzt der Unterschied, daB nur gerade Werte die Zickzackpunkte besetzen, während die ungeraden auf der Mittellinie liegen. Gemäß der gemachten Ableitung bedeutet also auch eine solche Form eine doppelte Oszillation. Eine derartige einfachere Figur von Doppeloszillation enthält das Diagramm über das molekulare Drehungsvermögen der homologen Acyl-1-Äpfelsäureäthylester in der Arbeit von H. ReItrer. ${ }^{2}$ ) Auch in diesem Diagramm ist die hineinzudenkende Verbindungslinie. zwischen dem zweiten und dem vierten $C$ besonders lang und Punkt 4 enthält, wie häufig einen Knickpunkt. Führt man in der oben angegebenen Weise die Linie auf eine, diese Besonderheiten ausschaltende Normalform zurück, so hat die vollkommen regelmäßige Doppeloszillationslinie (mit pendelnden ungeraden Werten) folgendes Aussehen:

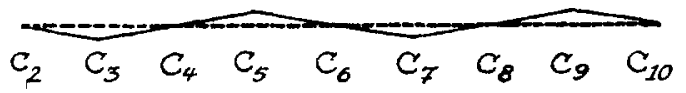

Fig. 7.

Der Unterschjed zwischen den Acyl-1-Mentholen und den Acyl1-Äpfelsäureäthylestern ist vom Standpunkt der schon orwähnten Tatsache aus, daß die zweibasischen Säuren der Fettreihe und ihre Ester bei den in diesem Abschnitt besprochenen physikalischen ÄuBerungen nur einfache Pendelung zeigen, recht beachtenswert. Denn auch die Äpfelsäureester enthalten zwei Carbäthoxylgruppen, unterscheiden sich aber von jenen durch ibren alkoholischen Hydroxylrest, an den sie noch Acylreste binden können. Man sieht, daB

punktierten Linie nur etwa ein Drittel von denen der oberen geraden $(4,8)$ von dieser betragen, und zweitens, daß das Verhältnis der Entfernungen der geraden Punkte von dem vorangehenden und von dem nachfolgenden ungeraden sich allmählich umkehrt. Nimmt man binzu, daß jede der beiden hinzuzudenkenden Mittellinien auch noch ihre besondere Form haben, so kann man aus alledem schließen, daß wenigstens vier verschiedene Faktoren die Lage der Konstanten der Fettsäuredissoziation bestimmen. Es wäre von größtem Interesse, den genauen weiteren Verlauf der Werte kennen zu lernen. Das gleiche gilt auch von der optischen Drehung der Acyl-l-menthole und dem anscheinend analogen Diagramm der bisher leider nur kurzen Reihe der Acyl-l-borneole (TschtGarFT). Vgl, das Diagramm in der Arbeit von H. RertTkR (folgende Anmerkung).

1) Z. 1. physik. Chem. 36 (1901), 164. 
ebenso, wie die Acyläpfelsäureester konstitutionell gewissermaßen zwischen den Estern der ein- und zweibasischen Säuren auf diese Weise stehen, so sie dies auch hinsichtlich der Form ihrer Oszillation tun. Diese ist tatsächlich eine doppelte, hat aber das Aussehen einer einfachen.

Dagegen zeigt die Reihe der Dissoziationskonstanten der Alkylmalonsäuren ${ }^{1}$ ), die man auch als Kombination einer zwei- mit einer einbasischen Fettsäure betrachten könnte, einfaches Pendeln.

Ansätze zur Doppeloszillation zeigen teilweise auch die Diagramme der von Pickard und Kenyon ${ }^{2}$ ) auf ihr Drehungsvermögen hin untersuchten homologen Fettsäureester der Carbinole $\mathrm{CH}_{3} . \mathrm{CH}(\mathrm{OH}) . \mathrm{R}$. Leider konnten sie indessen als zuverlässiges Beweismaterial hier nicht Verwertung finden, weil anscheinend der für die sichere Feststellung dieser Erscheinung erforderliche hohe Grad von Genauigkeit bei den Messungen nicht erreicht worden zu sein scheint.

Im übrigen fanden sich nirgends sonst Anzeichen von Doppelpendelung; nach meinen bisherigen Beobachtungen ist sie, wie gesagt, auf die einbasischen normalen Fettsäuren und einige ihrer A bkömmlinge beschränkt. Beispiele einfachen Wechselns der Dissoziations- und Drehungswerte im AnschluB hieran noch besonders aufzuführen, davon glaube ich absehen zu dürfen, indem auf solche Reihen schon von anderer Seite hingewiesen worden ist.

Nun zur Frage nach dem Sinne der Doppeloszillation! Hier ist erstens der Gegensatz zwischen den ein- und den zweibasischen normalen Fettsäuren in die Augen springend, und zweitens dessen Beschränkung auf die Dissoziationskonstante und die bei diesem Erscheinungsgebiete mit ihr in sichtbarer Beziehung stehende molekulare Drehung.

Die einbasischen Säuren unterscheiden sich von den zweibasischen, in denen sämtliche verfügbaren Valenzen, nämlich die Mehrheit der Valenzen der beiden endständigen Kohlenstoffatome, an Sauerstoff gebunden ist, dadurch, da B bei ihhen auf der einenSeite die Mehrheit an Wasserstoff geknüpft ist. Diese Mehrheit ist für die Doppeloszillation verantwortlich zu machen. ${ }^{3}$ ) Denn, wenn eines der Wasserstoffatome z. B. durch Halogen ersetzt wird, hat man

1) A. a. 0 .

2) Journ. Chem. Soe. 1914, I, 830.

3) Über die Bedeutung der polaren Mehrheit für die Benzolorientierung vgl. meine Ausführungen Journ. prakt. Chem. 98 (1918), 131. 
kein Anzeichen mehr für doppelte Pendelung. Die Reịhen fallen dann stetig ab, sie steigen nirgends auf, wie folgende kleine Tabelle der Dissoziationskonstanten von $a$ - bis 8 -Halogenfettsäuren ersehen läBt.

$-\mathrm{CH}_{9} \mathrm{COOH}$
$-\mathrm{C}_{2} \mathrm{H}_{4} \mathrm{COOH}$
$-\mathrm{C}_{3} \mathrm{H}_{6} \mathrm{COOH}$
$-\mathrm{C}_{8} \mathrm{H}_{8} \mathrm{COOH}$

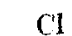

$1,55 \cdot 10^{-3}$
$8,59 \cdot 10^{-5}$
$3,0 \cdot 10^{-5}$
$2,04 \cdot 10^{-5}$

$\mathrm{Br}$

$1,38 \cdot 10^{-3}$

$9,8 \cdot 10^{-5}$

$2,6 \cdot 10^{-5}$

$1,91 \cdot 10^{-5}$
$J$

$0,7 \cdot 10^{-3}$

$9,0 \cdot 10^{-5}$

$2,3 \cdot 10^{-5}$

$1,71 \cdot 10^{-5}$

Wenn somit ein einziges Wasserstoffatom schon einen solchen Einfluß auf die Polarität von Säuren ausübt, so kann ich mir nicht vorstellen, wie eine elektronentheoretische Ableitung richtig sein kann, bei der Methylkohlenstoffatome ohne jede besondere polare Ursache sowohl mit der Ladung $\mathrm{H}+\frac{\mathrm{H}}{\frac{\mathrm{C}}{\mathrm{C}}}-$, als auch $\mathrm{H}+-\frac{\mathrm{H}}{\mathrm{H}}+\frac{\frac{ \pm}{\mathrm{C}}}{\mathrm{H}}+$ vorkommen können, wie es nach der Grundvorstellung von E. J. CuY z. B. im Äthan der Fall ist. Denn was bedeutet dann noch das dritte Wasserstoffatom für die polare Lockerung des am anderen Ende der Kette befindlichen Wasserstoffions in Säuren, wenn schon das eigne Kohlenstoffatom belic big positive oder negative Ladung tragen kann nur auf Grund seìnes bezüglichen Platzes in der Kette? Das rein schematische Abwechselnlassen der Vorzeichen zwingt E. J. Cuy, um mit seinen eigenen Worten ${ }^{1}$ ) zu sprechen, ,alle diese verschiedenen Fälle auf ein und dieselbe Ursache zurückzuführen" und man könnte ihm angesichts der Erscheinung der eingangs angeführten sehr ähnlichen schmelzpunktsoszillationen polar durchaus heterogener homologer Reihen und der Doppelpendelung sein Gebot vorhalten. da.B ,eine Theorie, die einen Fall erklären will, auch imstande sein muß, eine Erklärung für alle hier angeführten Fälle zu geben“. Ich bin der Meinung, da $B$ der Schematismus der Ableitung E. J.CUYs zu weit geht. $\left.{ }^{2}\right)$ Dennoch steckt etwas Richtiges darin, und man mag mitunter seine Schreibweise, ähnlich der VorLäNDERs, und unter gewissen Voraussetzungen als vereinfachten Ersatz für eine zwar schwierigere,

1) In der deutschen Übersetzung, 1. c.

2) Den gleichen Fehler, die zwar häufig, aber nicht immer anwendbare, polar abwechselnd sich fortsetzende Ladung der Atomketten zum Grundprinzip ibrer Theorie erhoben zu haben, machen FALK und NuLson [J. Amer. Chem. Soc. 32 (1910), 1637; Journ. prakt. Chem. 88 (1913), 97], weshalb auch ihre theoretische Erklärung der Oszillationen, wie schon bemerkt, nicht genügen kann. 
aber die Einzelheiten näher begründende theoretische Ableitung nach J. Stark verwenden können.

Im Falle der Oszillationserscheinungen der normalen zweibasischen Säuren scheint mir die polare Ableitung der Oszillation, obwohl sich wechselnde Polarität in den zwei Reihen der Dissoziationskonstanten unverkennbar mit ungerader und gerader Kettengliederzahl zeigt, nicht angebracht. Die Molekel der normalen zweibasischen Säuren sind strukturell völlig symmetrisch gebaut analog den gesättigten normalen Kohlenwasserstoffen; es findet sich nirgenda ein Atom oder ejne Atomgruppe, die infolge ihrer Besonderheit Anlaß zu polaren Betrachtungen bezüglich der Gesamtkette gäbe.1)

Dagegen würden die Erfahrungen über die Dissoziation stereo. chemisch untereinander verschiedener Säuren ohne weiteres berechtigen, auch bei den zweibasischen säuren einen in dem früheren Sinne sterisch verschiedenen Bau der gerad- von den ungeradzahligen als Ursache verschieden starker Dissoziation anzunehmen, indem nämlich die ersteren ihre Carboxyle in einer Art von anti-, die letzteren sie in einer Art von syn-Stellung trügen. Übereinstimmend mit unseren Fällen könnte man so z. B. die Hexahydrophtal- und -terephtalsäuren anführen, bei denen die trans-Verbindungen größere Dissoziationskonstante (und zugleich höheren Schmelzpunkt) aufweisen, als die cis-Verbindungen. ${ }^{2}$ )

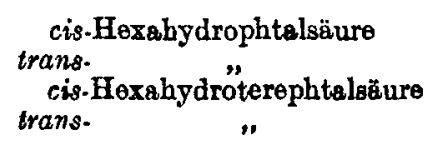

Diss.-Konst.
$4,4 \cdot 10^{-5}$
$6,2 \cdot 10^{-5}$
$3,0 \cdot 10^{-5}$
$4,6 \cdot 10^{-8}$

$\left[\begin{array}{c}\text { Schmp. } \\ 182^{\circ} \\ 215^{\circ} \\ 161^{\circ} \\ 200^{\circ}\end{array}\right]$

Ganz anders nun bei den einbasischen Säuren. Hier haben wir zunächst auch räumliche Verschiedenheit, wie bei den zweibasischen, infolge des Baues der Kohlenwasserstoffkette; dazu kommt aber nun eine polare Wirkung etwa in dem Sinne, wie CuY sie ableiten würde. Daher die Doppeloszillation! Bei den einbasischen haben wir ja an dem einen Ende der Kohlenstofflette drei Valenzen, d.h. die Mehrheit an Wasserstoff, und an dem anderen Ende wjederum die

1) Die Tatsache, der ungleichen Dissoziation der beiden Carboxyle bedarf keiner konstitutionellen Begründung zu ihrer Erklärung.

2) Die Verhältnisse wechseln sehr bei den Stereoisomeren, so daß man oft auch das Gegenteil findet, wie bei den erwähnten Säuren. Verschiedenheit hinsichtlich der Dissoziationskonstante scheint aber immer vorhanden zu sein. (Wie wollte man hier zur Erklärung schematisch abwechselede Ladung der Atome verwenden? Es kann doch nur räumlich bedingte Nähewirkung herangezogen werden.)

Z. enorg. u. allg. Clear. Bu. 116, 
Mehrheit an Sauerstoff gebunden, also an polare Antipoden. Das macht die Kette der Fettsäuren zu dem, was der Physiker einen Dipol nennen würde. Man kann eine solche Kette in lauter Dipole aus je zwei Kettengliedern sich zerlegt denken. Da aber die ungeraden immer ein überschüssiges Glied enthalten. so führt das notgedrungen zu zwei verschiedenen Reihen, einer, in der die Dipolbildung glatt aufgeht, und einer, in der sie nicht aufgeht. Daß die ersteren, nämlich die geraden, die stärkeren Säuren sein werden, liegt auf der Hand; denn bei den ungeraden $m u B$ die Fortsetzung der abwechselnden Ladung an den Enden zu Abschwächungen führen. Man kann das am einfachsten unter Anlehnung an die Schreibweise Vortändersi) an folgendem Schema zeigen:

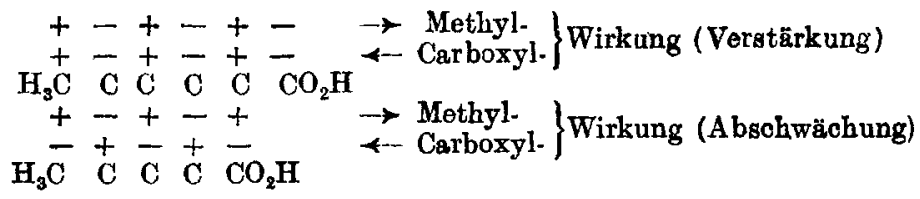

Natürlich findet im zweiten Falle keine völlige polare Neutralisation statt, wie es nach der rohen Schreibweise scheinen könnte, sondern nur Abschwächung; das Methyl ist gegenüber dem Carboxyl in seiner polaren Beeinflussung zu schwach.

Wie aber die beiden Effekte, der polar-sterische und der rein polare zusammenwirken, um die doppelte Oszillation der Konstanten homologer Fettsäurereihen zu bewirken, darüber kann man sich verschiedene Bilder machen; doch würde es angesichts der Unklarheit, welches wohl von ihnen mit den tatsächlichen Verhältnissen sich wirklich decken möchte, müßig sein, hier alle Möglichkeiten zu erschöpfen.

Deswegen will ich mich darauf beschränken, nur die mir als die einfachste erscheinende Erklärung darzulegen auf die Gefahr hin, nicht unbedingt die wahrscheinlichste gewählt zu haben.

Stellt man eine Dipolreihe einer Fettsäure in der Form der sterischen Fig. 2 (S.279) dar, so sieht man, daß auf der einen (unteren) Seite immer positive, auf der anderen (oberen) immer negative Vorzeichen liegen.

III.

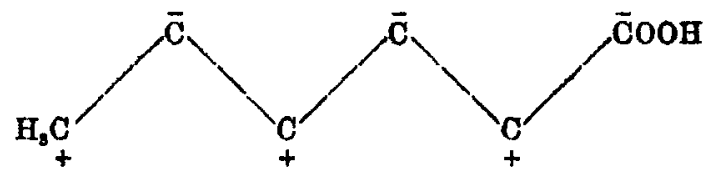

1) Ber. 52 (1919), 263. Die Vorgänge lassen sich valenzelektronisch näher begründen in der Art, wie ich das bei anderer Gelegenbeit [Ann. d. Chem. 888 (1911), 254] gezeigt habe. 
Während diese Zickzackform, solange nicht die Dipolerscheinung bestand, die an sich, wie früher dargelegt, polar-sterisch am meisten begünstigt war, ist sie es jetzt nicht mehr, indem zwischen den auf einer Seite gelegenen Vorzeichen kein Abwechseln der Ladung sich abspielt. Das findet nur statt, wenn die Drehung jedes zweiten Winkelstücks um $180^{\circ}$ erfolgt, so daß die ebenfalls früher dargestellte, aber als an sich weniger begünstigt befundene Fig. 3 (S. 279) entstebt:

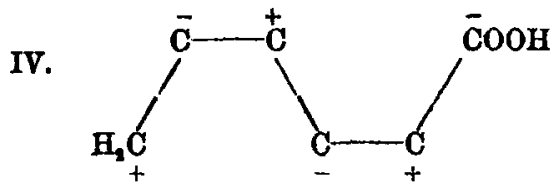

So wird diese Form nun die polar nach jeder Richtung hin am meisten begünstigste. Da B sie die Grundform der in Fig. 6a (S. 285) dargestellten normalisierten Doppeloszillation ist, wird man inzwischen wohl auch gesehen haben.

Die Heranziehung räumlicher Betrachtungen in dieser $\mathrm{Be}-$ sprechung zur Erklärung der behandelten RegelmäBigkeiten mag im ersten Augenblick vielleicht etwas fremdartig erscheinen. Wer aber die Benützung polarer Vorstellungen als berechtigt anerkennt, wird zugeben müssen, da $B$ diese Ableitung auf keinem unsichereren Boden steht, als jede andere stereochemische. Gewiß sind solche răumliche Gebilde, wie sie hier vorkommen, labiler, als andere, räumlich bestimmter konfigurierte, aber Labilität bedeutet doch noch keineswegs unbegrenzte Beweglichkeit. Die Anhänger der alten LAARschen Schwingungshypothese, nach der das labile Wasserstoffatom in Tautomeren beliebig und ständig hinundherschwinge, haben auf Grund der neuesten Forschungen auch die Erfahrung machen müssen, daß labile Zustände mitunter merkwürdig stabil sind und nur träge sich ändern. Warum sollten räumliche Konfigurationen bestrebt sein, sich in weniger begünstigte Lagen häufiger zu begeben, wenn sie einmal die meist begünstigte erreicht baben?

Wirrburg, im Juni 1921.

Bei der Redaktion eingegangen am 29. Juni 1921. 